\title{
VERS LA FIN DES BIDONVILLES À LISBONNE
}

\author{
DOMINIQUE CROZAT ${ }^{1}$
}

Résumé - Une des images de Lisbonne est celle d'une ville pauvre, dégradée, aux nombreux bidonvilles. Cela correspond à une réalité mise en valeur par les géographes au début des années 80. Mais aujourd'hui? Une étude des données disponibles et des politiques urbaines engagées montre au contraire une évolution rapide qui devrait, d'ici la fin du siècle, en faire une ville proche de la plupart des autres métropoles européennes.

Mots-clés: Bidonvilles, rénovation, politiques urbaines, changement urbain, Lisbonne.

Resumo - DESAPARECIMENTO DE BAIRROS DE LATA EM LiSBOA. Algumas características de Lisboa poderão conferir-lhe uma imagem de cidade pobre, degradada, com numerosos bairros da lata. Essa imagem correspondeu a uma realidade salientada pelos geógrafos no começo da década de 80 . Mas qual é a situação objectiva nos dias de hoje? Um estudo dos dados disponíveis e das políticas urbanas mostra uma evolução rápida que deveria fazer de Lisboa, até ao fim do século, uma cidade com características próximas da maior parte das outras metrópoles europeias.

Palavras-chave: Barracas/Bairros de latas, políticas urbanas, renovação, modificaciones urbanísticas, Lisboa.

Pendant longtemps le visiteur a emporté de Lisbonne l'image d'une ville figée dans son passé, ville grise, ville dégradée, ville de bidonvilles, les bairros de lata et leurs barracas: “... Les quartiers dégradés de la vieille ville, où des palais magnifiques mais vétustes sont occupés par plusieurs dizaines de familles, et les nombreuses constructions provisoires qui encombrent les cours des immeubles." (DRAIN, 1990) Certes, la réalité de cet habitat précaire n'est pas toujours merveilleuse, loin s'en faut; mais un regard trop pessimiste serait injuste pour une ville qui fait de gros efforts pour évoluer. L'étude qui suit s'efforce de montrer que la réalité est décalée de cette image.

\footnotetext{
1 Chargé de cours à l'Université du Mirail (Toulouse). Fax: (33) 05615044 41. Cet article est tiré d'un travail en cours qui vise à réaliser un atlas commenté de Lisbonne.
} 
Nous allons nous efforcer de dresser un état des lieux alors que la ville voit sa situation changer rapidement et présenter les conditions même de ce changement. Nous nous intéresserons à l'habitat précaire, plus particulièrement aux bidonvilles. Il ne s'agit pas de présenter l'ensemble des politiques d'urbanisme engagées à Lisbonne mais de montrer que cette évolution s'inscrit dans un contexte historique, urbanistique et surtout politique bien plus large.

Les bidonvilles commencent à apparaître comme une étape transitoire dans l'évolution de la ville. Pour des raisons propres à Lisbonne qu'il s'agira d'évoquer, cette situation a connu une ampleur, spatiale et temporelle, importante. Aujourd'hui, malgré un premier recul, les traces en sont encore visibles. Pourtant, un vaste mouvement de réhabilitation et de rénovation amène à évoquer la fin probable de ces problèmes: l'avenir de Lisbonne est celui d'une métropole européenne comme les autres.

Avant d'envisager étudier la situation présente, il est nécessaire d'évaluer l'ampleur du problème puis poser le problème de la terminologie. Il apparaît que la définition des bidonvilles est moins simple qu'il n'y paraît au premier abord et que par conséquent les solutions à apporter à ce problèmes seront tout aussi complexes.

\section{I - UN LOURD HÉRITAGE}

Il faut se souvenir en effet que la réalité fut longtemps peu flatteuse. Pour cela, on peut citer la présentation du problème par Daveau (1989, p. 898-899): "Em 1977 avaliavam-se em 110.000 as residências de construção não legal, sobre um total nacional de 2.165.000 fogos, com 90.500 daquelas nos distritos de Lisboa e Setúbal. Mas nova avaliação (BRUNO SOARES, 1984), referente a 1981, admite que se tenham então construído em Portugal, desde 1970, cerca de 270.000 fogos não licenciados, ou seja 42 por cento do total, chegando a proporção a ultrapassar 50 por cento em certos lugares da Área Metropolitana de Lisboa, no concelho de Loures, em especial. Não parece que o número de casas clandestinas esteja recentemente a diminuir, ainda que se destrua de vez em quando um ou outro núcleo de barracas que impedem a abertura de novas vias circulares ou de acesso às cidades, ou uma série de casas clandestinas estabelecidas em áreas litorais consideradas turísticas, quando e onde estas são cobiçadas por promotores com forte capacidade financeira e influência política. $\mathrm{O}$ alastrar dos bairros improvisados, enormes consumidores de espaço, de plano monótono e desprovido de arranjo funcional, que se torna difícil e caro prover a posteriori das necessárias infraestruturas, parece em muitos casos um facto irreversível. É em especial à volta de Lisboa [...] que o problema adquiriu maior importância. Os bairros clandestinos cobriam em 1985 cerca de $150 \mathrm{~km}^{2}$ e albergavam à volta de 300.000 pessoas na Área Metropolitana de Lisboa."

La carte proposée par DRAIN (1990, p. 285, reprise simplifiée: fig. 1) présente l'intérêt de distinguer urbanisation clandestine et barracas. Restons cependant prudents: c'est une réalité mouvante et, à terme, amenée à disparaître. Par ailleurs, 
sur le terrain, la distinction entre les deux est rarement aussi nette.

Si bon nombre de grands ensembles de la périphérie sont alors encore clandestins et se distinguent des cabanes, l'évolution de certaines de ces dernières, les plus anciennes, progressivement consolidées et agrandies, les rend parfois difficiles à repérer.

Surtout, l'auteur oppose les zones d'urbanisation clandestine qui seraient concentrées dans la périphérie proche du concelho de Lisbonne, au point d'en dessiner les limites, et celles de bidonvilles, exclusivement situés à l'intérieur. Cette vision trop réductrice doit être nuancée: ainsi à Sacavém, concelho de Loures, les barracas n'existeraient pas. Cette carte pourrait aussi donner l'impression fausse que la périphérie suburbaine est dominée par les seules constructions clandestines en oubliant l'importance de la construction légale relativement récente.

On y découvre enfin que la surface des zones d'urbanisation clandestine était, à la fin des années 80 , supérieure à celle du bâti ancien et légalisé. On comprend qu'on ait pu qualifier ces formes d'urbanisation (urbanisation clandestine et bidonvilles) de dévoreuses d'espace et évoquer des valeurs aussi importantes qu'à la fin de la citation précédente qui représentent deux fois la surface du concelho de Lisbonne, avec cependant une population proportionnellement moindre du fait des faibles densités d'une partie de ces emprises. A travers ces deux citations, le texte comme la carte, on mesure à quel point la tâche se révélait difficile.

\section{II - DES CAUSES MULTIPLES ET AGGRAVANTES}

Lisbonne se signale par la multiplicité des problèmes qui la submergent durant trente ans et expliquent l'accumulation d'un énorme retard en matière d'urbanisme: afflux de population exceptionnel, blocages légaux et économiques, situation d'urgence, laisser-faire et confusion politiques.

Lisbonne est la principale bénéficiaire de l'exode rural (DAVEAU, 1989, p. 793-813), surtout à partir des années 50. Comme partout en pareil cas, cela provoque des tensions sur le marché immobilier. Conjugué à un accroissement naturel qui ne décroît que tardivement, cela explique l'énorme accroissement de la population: c'est particulièrement sensible dans les concelhos périphériques où les recensements signalent presque partout des hausses de 30 à $60 \%$ de la population en dix ans, parfois même son doublement (FONSECA, 1988; RODRIGUES, 1994). De 1950 à 1970, l'aire urbaine de Lisbonne passe de 14 à $21 \%$ de la population totale du pays (SALGUEIRO, 1977).

Par ailleurs, l'afflux massif d'une population désargentée durant les années 70-80 (retornados) va renforcer brutalement le problème. Depuis, ce double mouvement a été relayé, mais à un niveau bien moindre, par l'immigration étrangère en provenance surtout des anciennes colonies. 


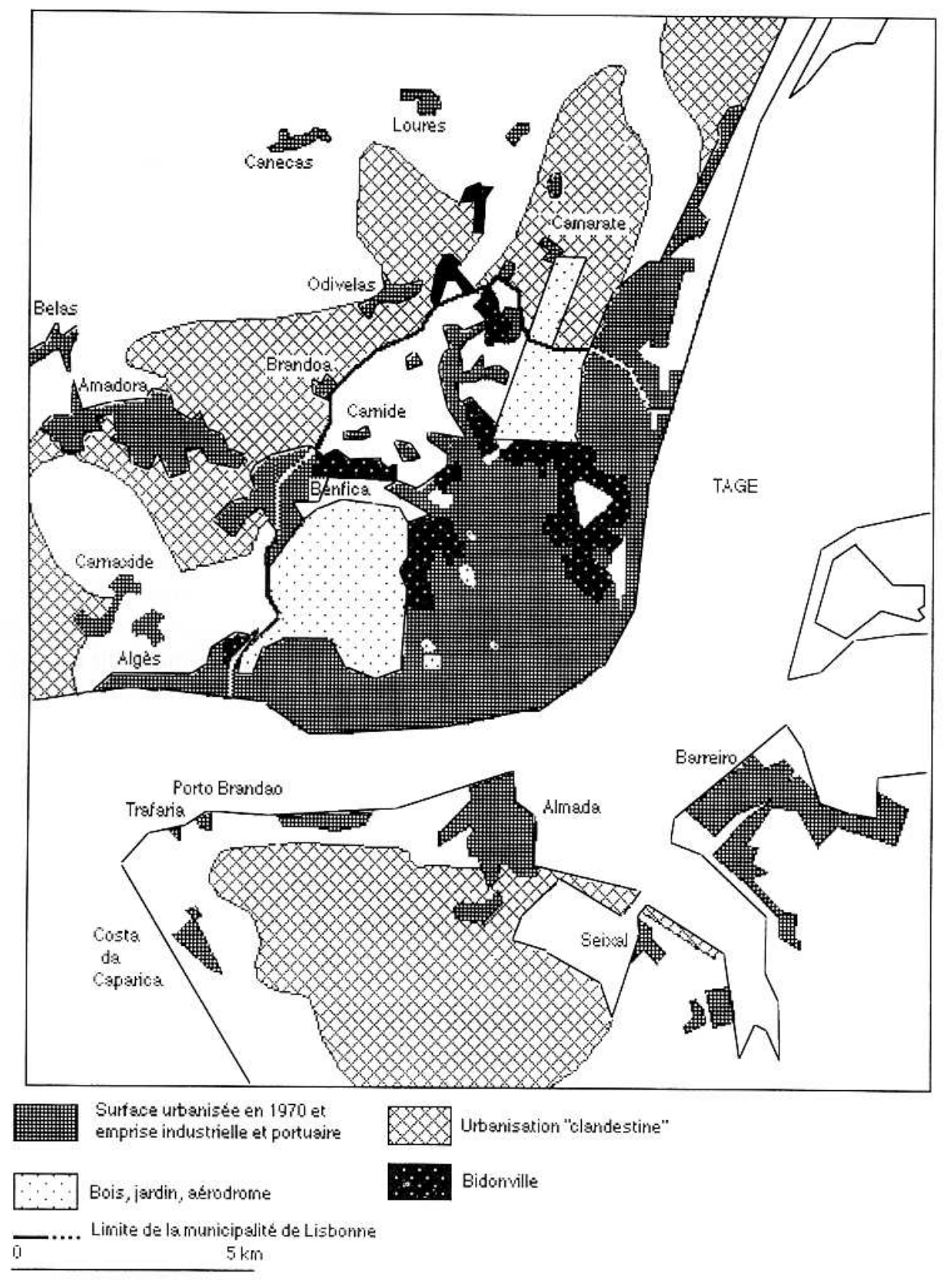

Fig. 1 - Les utilisations de l'espace à Lisbonne (d'après DRAIN, 1989, simplifiée)

Fig. 1 - A ocupação do solo em Lisboa (segundo DRAIN, 1989, simplificado) 
Mais la situation ne serait pas aussi grave sans la conjonction de problèmes urbanistiques: du coté du bâti existant, le blocage des loyers depuis 1938 a découragé toute vélléité d'entretien et de modernisation chez de nombreux propriétaires, y compris le plus important d'entre eux, la Câmara Municipal de Lisboa. Par la suite, la guerre coloniale impose d'autres préoccupations et détourne une part importante des moyens économiques.

Enfin, depuis vingt ans, l'évolution de la ville fait émerger un concurrent au logement bon marché: la construction privée se concentre surtout sur les investissements dans les bureaux et le logement de luxe, restreignant encore les disponibilités et augmentant les prix de l'immobilier. Au centre d'abord, ce mouvement s'étend progressivement à une grande partie de l'aire urbaine (SALGUEIRO, 1994).

La conjonction de cette explosion démographique, de la pauvreté, d'un investissement réduit des agents économiques, tandis que l'action des pouvoirs publics restait limitée, explique donc la gravité de la situation. A la dégradation de l'habitat ancien s'est ajoutée, sous l'effet de la pénurie de logements bon marché que plus personne ne voulait construire (SALGUEIRO, 1977), une multiplication des bidonvilles qui cependant existaient déjà: malgré tous ses discours populistes, la dictature n'avait pas eu une action très dynamique dans ce domaine mais le nouveau régime dans les années qui suivent la Révolution ne fait guère mieux.

Il se développe un marché immobilier des terrains clandestins, parallèle mais cohérent avec l'ensemble du marché immobilier légal car appuyé sur un détournement spéculatif des plans d'urbanisme (SALGUEIRO, 1977; DRAIN, 1990, p. 285). Après le 25 avril 74, une plus grande tolérance autant que le manque de moyens permettent un essor important de ce type de construction.

Dégradations et bidonvilles donnent alors de la ville cette image peu flatteuse évoquée plus haut: "au début des années 80 , on estimait à 100.000 le nombre des constructions illégales et au double celui des logements insalubres" (GUICHARD, 1990). Il faut se souvenir que $42 \%$ de la construction des années 70 sur l'ensemble du pays aurait été informelle, et que les deux tiers des édifices construits l'ont été dans les agglomérations de Lisbonne et Porto (LOBO, 1986), surtout Lisbonne. Cela devient le mode le plus fréquent de construction, on le normalise: d'un recensement à l'autre, une partie des logements clandestins, peu à peu viabilisés, sont comptabilisés comme des logements classiques (LOBO, 1986): "Não são raros os casos em que foi necessário elaborar planos de urbanização com vista à legalização e integração definitiva dos ditos bairros na cidade" (SALGUEIRO, 1977, p. 46).

Malgré d'épisodiques campagnes de presse, on constate une certaine tolérance des autorités qui amène à ne pas trop en parler afin de ne pas risquer de compromettre un système fragile et considéré comme nécessaire (SALGUEIRO, 1977). Une évolution n'est alors possible qu'à trois conditions: une reconnaissance politique préalable du problème, une évolution conjointe du marché immobilier et la possibilité de disposer des importants moyens nécessaires.

Si on compare l'attitude des autorités sur deux périodes, on voit ainsi que, 
durant les années 60-70, l'administration par son attitude très légaliste limite la construction de nouveaux quartiers d'habitat populaire qui aurait pu ainsi faire baisser les prix. En contrepartie, ce manque suscite la naissance de nombreux quartiers clandestins, et l'administration se montre assez permissive, fermant les yeux sur une réalité qui lui paraît satisfaitante. Par ailleurs le problème reste confiné au niveau local, sans publicité excessive: "Como os bairros clandestinos são "tolerados", em regra geral evita-se falar demasiado deles, na medida em que isso pode comprometer até o sistema que os gera" (SALGUEIRO, 1977). Il faut que s'écroule un immeuble de grande taille pour que le scandale ayant éclaté dans la presse, le Ministère de l'Intérieur diffuse une note puis commande une étude que d'ailleurs on se dépêche d'oublier une fois l'émotion publique retombée.

Vingt ans après, face à un problème proche ${ }^{2}$, la réaction est toute autre: les études scientifiques sont nombreuses, et surtout les études officielles ont précédé l'affaire ${ }^{3}$. Le discours change: le Président de la République dénonce le scandale de la situation, le Ministre des Travaux Publics explique qu'il est temps cette fois d'erradiquer les bidonvilles, ce qui jusqu'alors paraissait impossible 4 . On mesure le changement: ce qui était toléré comme désagréable, regrettable mais insurmontable apparait intolérable et alors tout devient possible.

Dans ces situations, la perception est aussi importante que la réalité. Ainsi, le changement de mode de production de l'espace passe nécessairement par une évolution préalable de son discours de valorisation (CASTELLS, 1981): la représentation du réel surdétermine l'action politique.

\section{III - IMAGE ET CONCEPTS}

Réglons d'abord le problème d'image évoqué plus haut. Il est certain que, pour des raisons liées à une évolution socio-économique menée selon des rythmes différents, du nord au sud de l'Europe, le langage commun n'a pas toujours les mêmes normes.

Au Portugal, on constate une ambiguïté dans la perception des quartiers de barracas: on les nie autant qu'on les assume. C'est ainsi le cas dans les plans vendus au public: aux cartes 4 et 5 du plus précis d'entre eux ${ }^{5}$, pourtant non touristique, on voit ainsi dans le quartier d'Ameixoeira deux rues où les cabanes, en cours de consolidation, sont figurées et un autre ensemble, dans la réalité bien plus pré-

\footnotetext{
2 Il s'agit de la Brandoa en février 69 et de ce que l'on a appelé «o caso do Lar Panorâmico» à Camarate, en janvier 93.

3 Ministério das Obras Públicas, Transportes e ComuniCaÇões (1990) - Características Recentes do Sector da Construção. Lisboa

Encontro Nacional da Habitação - Jornadas preparatórias. Lisboa, Abril 1992.

Livro Branco sobre a política da habitação em Portugal, Lisboa, Fevereiro 1993.

Et cette liste n'a pas la prétention d'être exhaustive...

${ }^{4}$ Diário de Notícias; j'ai relevé 6 mentions au printemps 93 et un rappel le17 juin 95.

${ }^{5}$ Guia Urbano. Forum Iberica, Lisbonne, non daté (mais récent), 99 pages, 107 feuillets cartographiques à l'échelle 1: 5.000 et 8 feuillets doubles à l'échelle 1: 1.500.
} 
caire, où seules sont représentées les voiries.

Par ailleurs, il s'opère aussi une distinction subtile entre groupes de maisons de transformation en habitat moins précaire ou leur population (GUERRA et al., 1995). Un habitant du nord de l'Europe va tout considérer comme des bidonvilles.

De plus, le terme, restreint à l'édifice lui-même, de barracas est souvent moins sévère qu'en français celui de bidonville. Mais il serait intéressant d'étudier l'évolution du vocabulaire spécialisé dans la presse portugaise depuis une quinzaine d'années: on y verrait probablement et assez logiquement un lent glissement susceptible de traduire l'évolution des modes de vie et des mentalités durant cette période.

Les définitions les plus complètes des termes employées sont données dans deux études (BRUNO SOARES, 1984; LOUREIRO DE MATOS, 1990) qui s'efforcent aussi de mettre en valeur les conditions de construction et l'état du marché immobilier des quartiers concernés.

On en retiendra d'abord une certaine profusion de termes: on parle de construction illégale, précaire, auto-construite, informelle, marginale ou clandestine. Ce dernier, le plus souvent retenu au Portugal, signifie effectuée "sem autorização das Camaras Municipais, às quais compete licenciar e controlar as obras realizadas por particulares" (BRUNO SOARES, 1994).

On a vu que l'aspect légal est en effet au Portugal plus important que dans des pays très pauvres où on préférera souvent d'autres adjectifs moins précis: "os termos marginal e/ou informal, englobam tanto o processo de construção de barracas como o de construções clandestinas. As primeiras são construções feitas geralmente com materiais velhos e usados, normalmente em madeira, com carácter precário, sobre terrenos públicos ou arrendados a particulares, constituindo aquilo a que vulgarmente se chama bairros de lata. Quanto às segundas, são construções de alvenaria de tipo e qualidade semelhantes às construções legais, sobre solos pertencentes aos ocupantes, adquiridos por este com o propósito de construção, constituindo, por vezes, “...conjuntos perfeitamente definidos, segregados das restantes malhas construídas..." (SALGUEIRO, 1972) - os bairros clandestinos" (LOUREIRO DE MATOS, 1990).

Si les premières trouvent d'abord leur origine dans la pauvreté, dans le cas des secondes ont voit primer les blocages structurels et l'organisation d'un marché spécifique, étudiés en détail par les auteurs cités plus haut. Cette distinction est ancienne puisque formulée au sujet de la Brandoa dès 1969 comme symbole de clandestinidade permitida (SALGUEIRO, 1977, p. 47). Deux remarques s'imposent cependant.

Tout d'abord, cette typologie précise ne résoud pas tous les problèmes car l'observateur, sur le terrain, ne leur donne pas toujours une traduction formelle: les quartiers clandestins sont souvent caractérisés par l'extrême variété de leurs constructions puisque les capacités d'investissement des populations concernées sont elle-mêmes extrêmement variables; s'y ajoute l'ancienneté, et donc l'importance des améliorations du quartier. Il n'est donc pas rare de trouver des maisons très précaires, habitat provisoire et proche dans la forme de celui des barracas, dans 
les lotissements clandestins les plus récents. A l'inverse, des ensembles précaires mais anciens et donc consolidés et améliorés ne ressemblent guère à des bidonvilles: ainsi à l'extrémité sud de la rue Sampaio Bruno, à proximité du cimetière des Prazeres. Il apparaîtra donc difficile, au paragraphe suivant, de les mettre en valeur à travers des séries statistiques claires et précises.

De fait, les choses se compliquent si on tient compte de deux remarques supplémentaires. Une partie des barracas sont construites avec des matériaux plus résistants que le bois, en dur, même si cela reste précaire. Cette différence entre le bidonville et les barracas est ancienne, étudiée par exemple à Barcelone dès les années 50 (GEORGE, 1961). De légale et économique, la distinction devient aussi formelle et temporelle: l'inscription plus ou moins ancienne dans le paysage qui va permettre de consolider la cabane rudimentaire des débuts pour la transformer peu à peu en une véritable maison selon un processus qui peut durer parfois des dizaines d'années. Cette idée est reprise par les statistiques portugaises qui distinguent barracas et casas de madeira e outros alojamentos improvisados.

Cela nous amène à la seconde remarque: quel critère sera alors pertinent pour appréhender la réalité des bidonvilles? Il semble que les modes de vie seront les meilleurs marqueurs: disposer de l'eau courante ou de l'électricité changera radicalement la vie des populations concernées. Ce critère permettra de distinguer les logements très précaires des autres.

Mais au-delà une autre frontière sociale se dessine qui nous complique un peu plus la tâche: dans un pays développé, la simple possession des fournitures de base (eau, électricité, égouts) permet-elle de disposer d'un niveau de confort moyen? Non, on y ajoutera le fait de disposer d'une salle de bain. Cela crée un second niveau de logements au confort insuffisant. Mais cela amène en conséquence à prendre en compte aussi l'habitat ancien dégradé. La remarque est d'autant plus justifiée qu'au Portugal, du fait du blocage des loyers pendant une très longue période, la situation était dans les années 80 particulièrement grave.

D'autre part, du fait de cette distinction, les politiques menées pour résoudre les problèmes seront de deux sortes (BASTIÉ et DÉZERT, 1991, p. 349). Tout d'abord, dans les quartiers anciens dégradés, on va opposer la rénovation avec aménagement des parties dégradées, destruction des îlots les plus insalubres (au nord de Graça, par exemple) et la restauration ou réhabilitation du cadre ancien (Alfama, Bairro Alto). Dans les quartiers de barracas on va pratiquer la rénovation en les détruisant pour reconstruire, généralement de grands ensembles d'habitation collectifs. On parlera d'une politique de relogement des habitants concernés.

Enfin, dans les zones d'urbanisation clandestine, souvent de bien meilleure qualité, se pose le problème du raccordement aux réseaux (eau, électricité, égouts, téléphone, voirie), dans l'ensemble presque terminé à Lisbonne, grâce à un effort important depuis le début des années 80: on verra apparaître ces quartiers comme plutôt mieux équipés que la moyenne. Le deuxième aspect, plus tardif, tient à la légalisation de ces lotissements. Démarré de manière systématique plus tardive- 
ment, ce processus est aussi en voie d'achèvement ${ }^{6}$ : bientôt, plus rien ne distinguera ces zones du reste de la ville si ce n'est leur manque d'unité urbanistique. Ceci explique qu'on se consacrera surtout à la mise en valeur des deux premiers types d'habitats (immeubles dégradés du centre ville et surtout barracas) dans les paragraphes qui suivent.

\section{IV - EVALUER LA QUALITÉ DES LOGEMENTS À LISBONNE}

On voit donc apparaître trois types d'habitat marginal. Si, malgré un essor durant les années 70, le premier type (les bidonvilles) reste relativement secondaire par rapport au nombre total de logements, le second (les constructions clandestines) a représenté une part notable des constructions de cette époque (LOBO, 1986). On a déjà signalé que ces dernières seront volontairement négligées sauf mention rapide lorsqu'il s'agira de montrer à la fin de cette étude que les efforts en direction de l'habitat précaire s'inscrivent dans un cadre plus large de modernisation rapide de la métropole lisboète.

Les premiers sont par contre très spectaculaires, surtout dans une ville européenne. Il reste à ajouter le troisième type: les immeubles anciens déteriorés. Distincts des barracas, ils n'en révèlent pas moins l'ampleur des efforts à accomplir. Identifier les uns et les autres pour en évaluer l'importance n'est pas très facile car les séries statistiques ne recouvrent pas toujours une réalité qu'on vient de présenter comme complexe.

Les données tirées des recensements de 1991 s'efforcent cependant de distinguer les barracas (construites en dur) des maisons de bois et autres logements improvisés: une vingtaine de milliers chacune pour l'ensemble du pays, chiffre assez stable de 1981 à 1991, dont 80\% dans les districts de Lisbonne et de Sétubal. L'étude du Ministère de l'Equipement Social de 1984 (MINISTÉRIO DO EQUIPAMENTO SOCIAL, 1981), au pire de la crise, prend ce type de données pour base.

Surtout, on a vu la difficulté à définir avec précision les différents types d'habitat avec un tel critère. Il apparaît donc préférable d'essayer d'abord de cerner le logement précaire par son niveau de confort car c'est le principal facteur susceptible de déterminer des modes de vie spécifiques aux populations concernées.

On représentera donc sur la figure 2 la part des logements ne disposant pas du confort de base (eau, électricité, égouts). C'est la principale caractéristique des logements très précaires: cela permettra donc de mettre en valeur les barracas et l'ensemble de la construction informelle recouverte en français par le terme courant de bidonville.

Au-delà de la satisfaction de besoins essentiels, dans un pays développé la possession d'une salle de bains est un autre marqueur important en matière de confort. C'est souvent une carence majeure des appartements dégradés du centre ville, bien qu'alors la précision de l'observation soit moindre puisqu' on trouvera aussi

6 Expresso, 17 Maio 1997, Cadastro único pronto em 1998. 
dans l'aire urbaine d'autres logements précaires bien que moins nombreux: barracas en cours de consolidation et d'amélioration, résidences secondaires. $\mathrm{Ce}$ manque de précision amène à privilégier une représentation globale des logements à problème en ajoutant les données utilisées sur la figure 2 plus les nouvelles données. On représentera l'ensemble sur la figure 3: part des logements ne disposant que d'un confort partiel (sans confort de base et/ou sans salle de bain). Il paraît en effet préférable d'ajouter les deux séries de données pour réaliser un bilan de l'habitat de mauvaise qualité.

Nous allons ensuite croiser cette première approche avec les données utilisées par les pouvoirs publics lors de la définition du PER (Plano Especial de Realojamento) que nous présenterons en détail dans la dernière partie de cet article. Ces données ont été fournies par l'IGAPHE (Instituto de Gestão e Alienação do Património do Estado) mais produites par l'INH (Instituto Nacional de Habitação) en 1994. Elles ne sont pas utilisées brutes mais rapportées au nombre total d'immeubles (figure 4) et à la population (figure 5) tels que révélés par le recensement de 1991, la relative marginalité du phénomène observé minorant d'autant la marge d'erreur due à l'évolution entre les deux relevés.

Il importe en effet de se préoccuper de la fiabilité des données utilisées; c'est même la raison de cette utilisation croisée. Les données tirées du recensement de 1991 (figures 2 et 3) sont probablement exagérées du fait d'une amélioration rapide depuis cette date. A l'inverse, pour des raisons de prestige, celles du PER sont sous-évaluées par certains concelhos notamment en périphérie (Palmela, Mafra) même si les conditions juridiques autant que l'ampleur de l'aide financière consentie ont incité la plupart d'entre eux à profiter de l'aubaine et ne pas trop minimiser la réalité. De plus, dans les périphéries les plus lointaines de l'aire urbaine, une très grande partie de l'habitat précaire n'est pas concernée par le PER puisqu'il s'agit de résidences secondaires.

Leur confrontation devrait permettre une évaluation assez précise; les écarts entre les deux séries de données restent limités. Mais les données concernant les logements dépourvus d'une partie ou de la totalité du confort de base (source INE, figure 2) permettent de mettre en valeur l'ensemble de la construction sauvage, sans distinguer les barracas des quintas devenues résidences secondaires, des cabanes à frites du bord de mer voire, mais c'est assez rare, des lotissements plus ou moins huppés ${ }^{7}$ non reliés aux réseaux publics mais dotés de manière autonome de ces services.

\footnotetext{
${ }^{7}$ Lagoa de Albufeira ou Cova do Vapor par exemple.
} 
En fait, cette imprécision se révèle peu gênante car mineure sur la majeure partie de l'agglomération, surtout son cœur plus dense qui nous intéresse le plus. Les écarts les plus importants, les seuls qui méritent qu'on s'y arrête, sont caractéristiques des périphéries plus lointaines où leur ampleur laisse supposer celle de ces constructions secondaires sans que, on vient de le voir, les deux se recoupent systématiquement.

\section{V - L'IMPORTANCE DES LOGEMENTS INCONFORTABLES}

Les logements partiellement ou totalement dépourvus de confort de base (figure 2) étaient en 1991 extrêmement nombreux: près de 80.000 , soit $8 \%$ du nombre total de logements dans l'aire métropolitaine, et des valeurs par concelho comprises entre 2,6 et $14,3 \%$. On peut opposer Lisbonne (4\% du bâti) à sa proche périphérie où les valeurs sont plus faibles du fait de la multiplication des grands ensembles et des villas, souvents récents et bien équipés: à Almada, Amadora, Cascais, Loures, Oeiras, Seixal, Sesimbra plus de la moitié des immeubles ont été construits après 1971; à Mafra, Moita, Palmela, Setúbal, Sintra et Vila Franca ce taux dépasse les $40 \%{ }^{8}$.

Surtout, ces taux s'accroissent beaucoup dans les périphéries plus lointaines: partout plus de 6,5\% des logements sont concernés. Les quintas mal équipées sont nombreuses, plus que les barracas, et les deux phénomènes s'additionnent sans se cotoyer. La confrontation avec d'autres données permet de préciser l'observation: la figure 4 laisse supposer que les premières sont les plus nombreuses. Ce qui explique partiellement l'important écart (plus de $50 \%$ ) constaté avec les données du recensement regroupées dans les rubriques barracas et maisons de bois et autres logements improvisés. Aussi, les concelhos de Sintra, de Loures et d'Almada, particulièrement concernés par ces caractéristiques, demanderaient une étude fine, par freguesia, afin d'expliquer leurs taux élevés (autour de 5\%). Dans les deux premiers la localisation des constructions est primordiale selon qu'elles sont situées dans l'immédiate proximité des zones denses ou, au contraire, éloignées du centre de l'agglomération. Mais même alors, la distinction resterait malaisée; la plage de Fonte da Telha (Almada) est caractéristique: entièrement constituée par des logements dans ce cas, les résidences permanentes y sont sensiblement aussi nombreuses que celles de villégiature.

\footnotetext{
8 Source: INE, Recenseamento Geral 1991.
} 


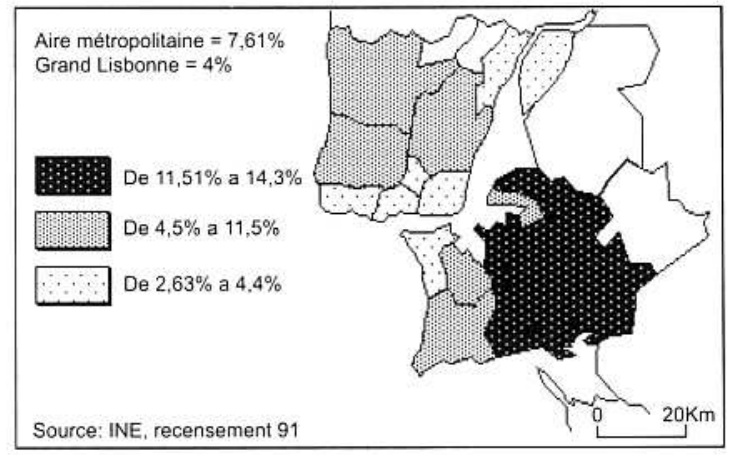

Fig. 2 - Logements dépourvus du confort de base

Fig. 2 - Percentagem de habitações desprovidas de água, electricidade e esgotos

Sur la figure 3 apparait la part des logements qui ne disposent pas de tout le confort: aux logements très précaires (figure 2 ), s'ajoutent ceux qui ne disposent pas de salle de bain. La différence entre cet indicateur et le précédent permet de cerner sans les distinguer, sinon par leur localisation, trois types de logements: les barracas en cours de consolidation, probablement les moins nombreuses, les immeubles dégradés du centre ville mais aussi les constructions rurales souvent bien plus médiocrement équipées.

Le résultat est fort honorable pour une métropole européenne pauvre: dans l'agglomération dense sauf à Lisbonne et Montijo, on a partout des taux d'équipement équivalents à ceux de la France (plus de 93\% des logements équipés)! Dans ce domaine, le progrès serait donc très net depuis 1981: le nombre de logements mal équipés chute de 200.000 à 120.000 alors que sur la même période le nombre total de logements augmente de près de $10 \% \ldots$

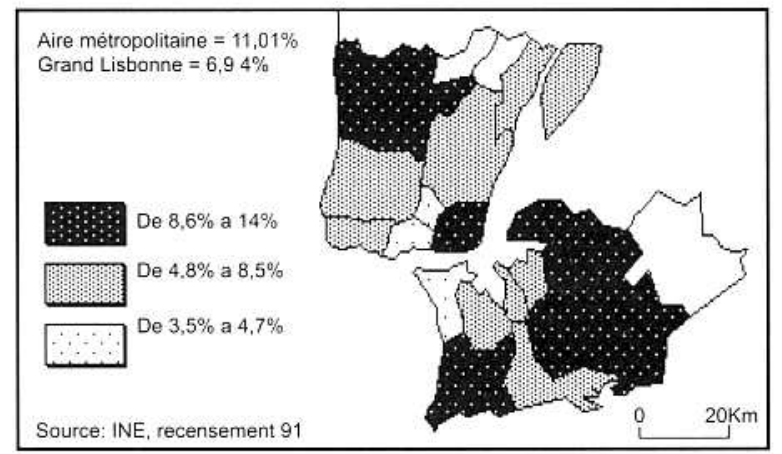

Fig. 3 - Logements inconfortables

Fig. 3 - Percentagem de habitações desconfortáveis

Il demeure qu'à Lisbonne, 9\% des logements ne sont pas équipés: c'est très 
important. Lisbonne est avec Setúbal le concelho de l'agglomération dense où, du fait de l'importance plus forte du bâti ancien, la part des logements mal équipés est assez logiquement la plus forte. On doit s'attendre dans une étude plus précise à une forte concentration de ces logements sous-équipés dans les freguesias du centre, d'habitat plus ancien, plus visibles, surtout des touristes, ce qui expliquerait le jugement pessimiste qu'ils emmènent sur la situation réelle de l'ensemble de la ville.

Dans la première périphérie, avec peu d'immeubles anciens, ces chiffres sont par contre excellents, à peine supérieurs à ceux des constructions sans confort de base. Bien sûr, les constructions légales récentes, majoritaires, sont bien équipées. On en déduit que le paysage présente de forts contrastes entre logements bien équipés et ceux totalement démunis. On voit ici nettement se différencier les barracas (figure 2) à l'intérieur d'un ensemble où l'urbanisation clandestine était importante jusqu'à une époque récente.

Cela illustre la nécessaire distinction introduite plus haut entre une définition légale (urbanisation clandestine) et sociale (les barracas). Une grande part de ces lotissements étant destinée aux classes moyennes, ils disposent d'un niveau de confort excellent. Une fois ceux-ci raccordés aux réseaux, ils n'apparaissent plus dans les données utilisées ici; leur problème est alors seulement légal (et fiscal). Leur normalisation bientôt achevée, ces quartiers ne se distingueront plus du reste de la ville.

Au-delà de cette première périphérie, les valeurs sont cependant beaucoup plus élevées: seuls 75 à $90 \%$ des logements sont équipés. Le très fort taux de constructions sans confort de base compte pour beaucoup certes, mais s'y ajoutent cependant 5 à $8 \%$ de logements partiellement équipés. C'est là, et seulement là, c'est à dire en lointaine périphérie suburbaine, voire semi-rurale, que l'on peut parler d'une ville médiocrement équipée encore que partout ces chiffres soient équivalents à ceux de la Grande-Bretagne (taux d'équipement de $77 \%$ ) et meilleurs que ceux dont pouvait se flatter la France en $1975(70,3 \%)^{9}$.

Les données fournies par l'IGAPHE relatives au PER (tableau 1; figures 4 et 5) complètent les données que nous venons d'utiliser: malgré quelques discordances (Amadora) les deux évaluations correspondent. Elles montrent l'extrême concentration du phénomène: un tiers des barracas de l'aire métropolitaine se concentrent encore à Lisbonne, un autre tiers dans les 3 concelhos qui la jouxtent (Amadora, Loures et Oeiras).

Mis en relation avec le parc immobilier (figure 4), le phénomène des barracas reste encore important dans l'agglomération dense. La même remarque s'impose si on s'efforce d'évaluer la population concernée (figure 5). Mais des progrès semblent avoir été accomplis depuis le début des années 80 et les données citées plus haut: deux fois moins de logements insalubres pour un parc plus important, ce n'est pas négligeable...

Tableau I - Le Programa Especial de Realojamento (P.E.R.)

${ }^{9}$ INSEE. Tableaux de l'économie française. Paris, ed. 95, p. 49 
Quadro I - O Programa Especial de Realojamento (P.E.R.)

\begin{tabular}{|l|c|c|}
\hline \multicolumn{1}{|c|}{ Concelhos } & Nb de barracas & Nb de fogos \\
\hline Lisbonne & 10000 & 11129 \\
Amadora & 4835 & 3495 \\
Loures & 3600 & 3904 \\
Oeiras & 3165 & 3169 \\
Almada & 1924 & 2156 \\
Cascais & 1361 & 2051 \\
Sintra & 1591 & 1591 \\
Setubal & 1121 & 1272 \\
Vila Franca de Xira & 765 & 765 \\
Seixal & 444 & 635 \\
Montijo & 307 & 293 \\
Barreiro & 250 & 230 \\
Moita & 127 & 160 \\
Sesimbra & 122 & 128 \\
Azambuja & 74 & 80 \\
Mafra & 62 & 62 \\
Palmela & 27 & 61 \\
Alcochete & 40 & 41 \\
\hline
\end{tabular}

Source: IGAPHE, 95

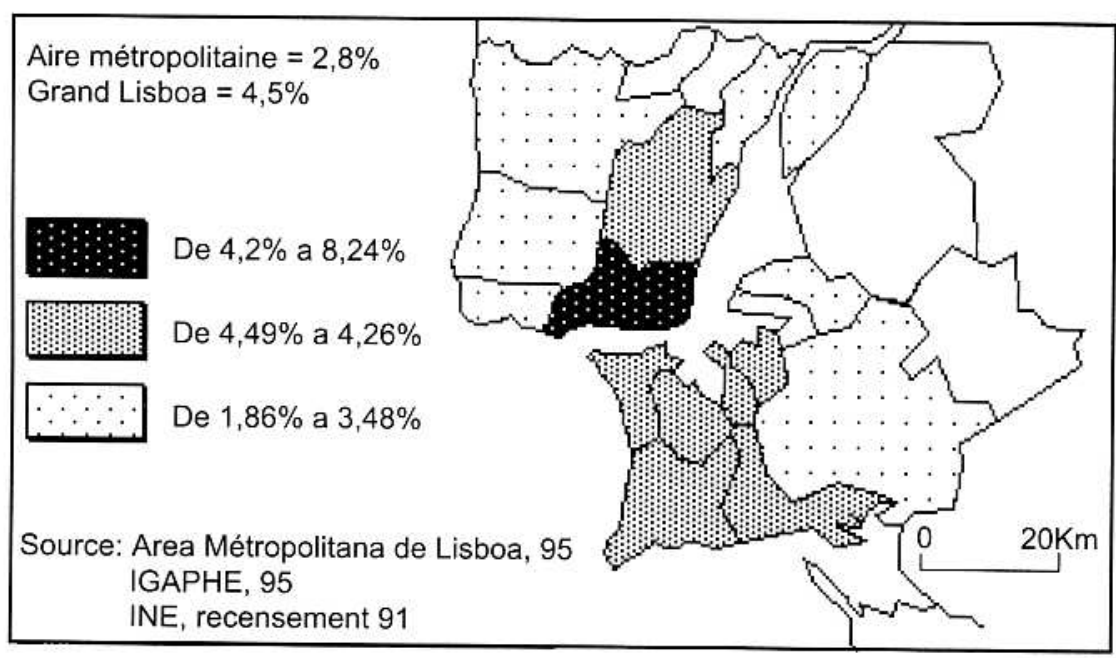

Fig. 4 - Evaluation de l'importance des barracas

Fig. 4 - Avaliação da importância das barracas

Au-delà, dans les périphéries plus lointaines, aux politiques urbaines moins volontaristes s'ajoutent une pression foncière moins forte, un niveau de vie plus faible et des résidences secondaires mal équipées fréquentes. Il s'agit maintenant 
de présenter les programmes d'action susceptibles de résoudre ces problèmes. Les progrès constatés depuis quinze ans devraient en effet s'accélérer.

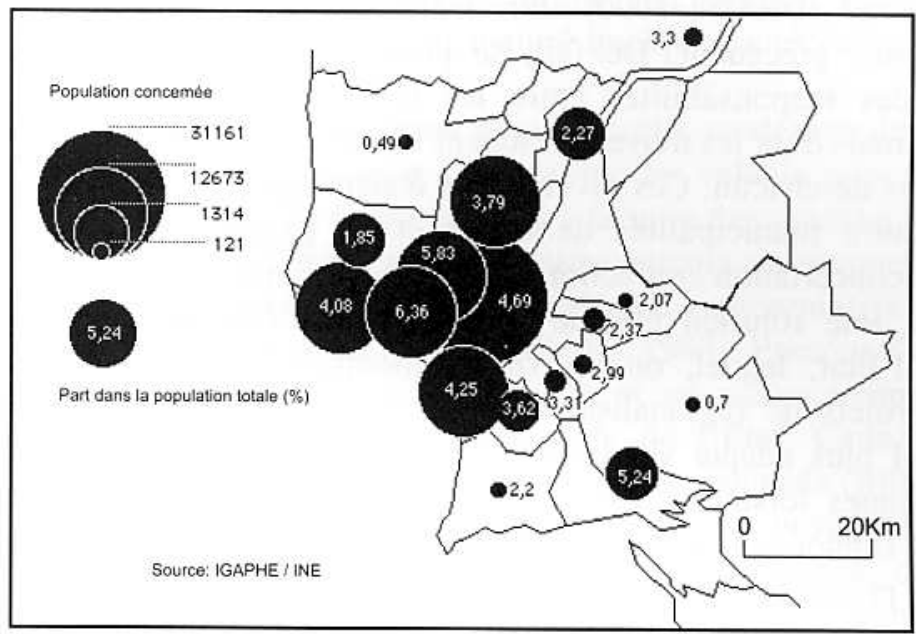

Fig. 5 - Evaluation des populations concernées par le P.E.R.

Fig. 5 - Avaliação da população abrangida pelo P.E.R.

\section{VI - UNE AMBITION URBANISTIQUE NOUVELLE}

Présenter et éventuellement évaluer une politique publique est partout difficile: aux spécificités locales s'ajoutent les incertitudes sur la réelle volonté d'aboutir des autorités, autant que celle de la longue durée nécessaire à ce type de programmes.

A Lisbonne, les premiers projets des années 70 et 80 , ont souvent été plus incantatoires qu'efficaces, autant par manque de moyens que de volonté (BRUNO SOARES, 1984). Ces lacunes avaient d'ailleurs amené après 1974 la constitution de coopératives, commissions d'habitants dont on a parfois exagéré l'importance: si 52 opérations furent effectivement engagées dans le district de Lisbonne bien peu purent être menées à terme. Puis la longue période libérale amena à privilégier les interventions privées y compris dans des domaines (le logement social) où les particuliers répugnent généralement à s'engager... L'ensemble de la politique urbaine était d'ailleurs marquée du sceau de la même orientation idéologique et l'idée d'une planification voire simplement d'un développement coordonné de la ville était taxé de gauchisme. Aussi, la ville centre semblait menacée d'implosion et perdait rapidement sa population (un recul de près de $18 \%$ entre 1981 et 1991). A la fin des années 70, la situation pouvait déjà apparaître comme grave. La décennie suivante fut marquée par l'immobilisme. Sans véritable projet urbanistique, l'équipe municipale s'appuyait sur des études vieillies pour annoncer des projets sans suite. Le projet de plan directeur de 1964, bien que 
révisé en 1972, était largement dépassé sans avoir jamais été vraiment mis en oeuvre ni même adopté (GUICHARD, 1990). On pouvait bien parler "d'urbanité sans urbanisme" (DRAIN, 1990) alors même que l'aire urbaine connaissait une croissance sans précédent. De fait, se posaient plusieurs problèmes: celui du découpage des responsabilités entre les différents concelhos aux préogatives importantes mais dont les moyens restaient limités face à la diversité et à la gravité des situations de chacun. Ces divergences étaient aggravées par les antagonismes politiques entre municipalités de droite et de gauche. La constitution d'une structure de concertation et d'action à l'échelle de l'aire métropolitaine s'imposait. Par ailleurs, une solution globale aurait mérité d'être mise en œuvre avec le soutien de l'Etat, lequel, on l'a vu, se montrait fort réservé, tandis que les différents projets de régionalisation éventuellement susceptibles de fournir un cadre spatial plus adapté s'enlisaient. Bref, on comprend que beaucoup soient restés sceptiques lorsqu'au début des années 90 furent mis en place plusieurs projets dont l'annonce apparaissait comme aussi tapageuse et creuse que ceux qui les avaient précédés. D'ailleurs leur préparation se révéla souvent longue et complexe afin de réunir des intervenants nombreux, aux objectifs et opinions forts divers. Il apparaît cependant, avec maintenant quelques années de recul, que, enfin, Lisbonne change (SALGUEIRO, 1994; CACHINHO, 1994) et très vite. C'est que deux facteurs essentiels sont rassemblés: volonté d'aboutir et moyens. On assiste en fait à la convergence d'efforts nombreux. Si l'initiative privée n'est pas négligeable, il convient cependant de ne pas en exagérer l'importance: elle est relativement marginalisée par les efforts publics et ne s'intègre que rarement, sous la contrainte réglementaire, sans beaucoup participer à la conception, dans cet effort de redéfinition urbanistique que nous présenterons ensuite. Elle peut cependant parfois se montrer spectaculaire, lorsque dans le cas de la constructions de centres commerciaux ${ }^{10}$, elle est concentrée sur un secteur. De par son origine on peut lui trouver deux formes: les sociétés spécialisées qui réalisent des projets importants (SALGUEIRO, 1994) dans l'immobilier destiné aux classes moyennes et le tertiaire, et les particuliers. Les premières s'intéressent peu au domaine que nous étudions ici, le plus souvent à l'occasion de conflits pour l'occupation de l'espace ${ }^{11}$; leurs investissements restent encore majoritairement limités à l'immobilier de luxe.

Dans le cas des seconds, l'enrichissement d'une partie de la société portugaise permet à des particuliers de plus en plus nombreux, d'acheter, de rénover ou améliorer leur logement. Ce mouvement, peu spectaculaire mais réellement important, devrait même s'amplifier si aboutissent les discussions ${ }^{12}$, pour modifier les bonifications d'emprunts. L'habitat ancien et dégradé comme bon nombre de constructions précaires devraient en profiter. Enfin, cela relaie et amplifie les initiatives d'origine publique.

\footnotetext{
10 Expresso, 24 Maio 97, p. 17

11 Correio da Manhã, 8 Outubro 94.

12 Expresso, 24 Maio 97, supl. Economia e negócios, p. 16.
} 
Dans leur cas, l'enjeu est triple: faire de Lisbonne une métropole moderne conçue dans sa globalité, reprendre le contrôle urbanistique de la ville et enfin réhabiliter le politique, légitimer l'idée d'une intervention publique dans ce domaine. $\mathrm{Vu}$ le contexte historique, et malgré quelques antécédents très anciens, c'est une innovation au Portugal.

Cette action passe par la mise en place d'outils modernes de planification. Dans le concelho de Lisbonne, mais la remarque est valable pour la plupart des communes de la métropole, ils furent adoptés à la suite de consultations très larges aussi bien des services concernés que des autres acteurs du secteur mais aussi la population ou les entités publiques comme privées représentatives (entreprises, organisations sociales, culturelles, etc...) (TOMAS, 1993). Prenons l'exemple de la ville-centre car il apparaît comme un modèle et en même temps il influence fortement l'action de ses voisins comme celle de l'Etat. Cette entreprise de planification y est structurée par une série de plans coordonnés (BRUNO SOARES, 1994): - Plano Estratégico de Lisboa (PEL, juin 92), plus prospectif, dont le rôle consiste à donner les grandes orientations: ouverture européenne, définition d'un projet collectif et cohérent, identification des priorités et des grandes initiatives telles que Expo'98 ou Lisboa Capital Europeia da Cultura 94. - Plano Director Municipal (PDM, septembre 93), élément régulateur du dispositif. Devenu obligatoire au Portugal, il correspond aux différents instruments d'administration urbanistique mis en place dans la plupart des pays européens. On attend de lui qu'il précise clairement les règles afin de maîtriser l'urbanisation mais aussi qu'il favorise une redynamisation de la ville. - Planos e Projectos Prioritários (PPP), structuration souple et ouverte qui permet un réajustement permanent des objectifs et des réalisations en s'adaptant à l'évolution des besoins (comme aux opportunités). Beaucoup plus concrets et très ciblés, ces plans offrent aussi la possibilité de concentrer des moyens importants sur des objectifs très délimités tout en coordonnant l'action de multiples intervenants, y compris extérieurs. Cela permet ainsi d'intégrer des plans de plus vaste envergure tels que le PER, présenté plus loin, tout en maintenant la cohérence de la politique menée au niveau local. L'ensemble de cette nouvelle planification se révèle très pragmatique. Son originalité et une part importante de ses premiers succès tient dans cette coordination entre éléments assez souples. Le retard important accumulé par rapport à d'autres pays européens explique pour une grande part ces caractéristiques: moins théorique et plus global que les politiques menées ailleurs, il bénéficie de l'expérience de leurs erreurs. S'y ajoutent des spécificités portugaises: le pouvoir important des câmaras municipais, antérieur à cet effort permet à celle de Lisbonne de structurer l'ensemble des politiques menées ainsi au plus près du terrain. Mais cela impose des contraintes plus grandes, qu'il s'agisse de l'ampleur de la tâche (le retard) ou la multiplication des intervenants: le poids relatif de Lisbonne a régressé durant cette longue période d'immobilisme, les autres concelhos se montrent donc plus sourcilleux sur le respect de leurs prérogatives. Il demeure que, malgré des frictions, la plupart des entités de la périphérie ont 
compris l'intérêt de la démarche et, dotées elles-même de PDM souvent convergents, profitent de l'effet d'entrainement induit par les changements à Lisbonne. Le même problème se retrouve au niveau des structures plus vastes, qu'il s'agisse des balbutiements de l'Área Metropolitana de Lisboa ou du regain d'intérêt de l'Etat pour ces questions, ce qui implique un interlocuteur certes souvent conciliant ${ }^{13}$ mais désireux aussi de garder un pouvoir de contrôle. Et comme ses financements se révèlent indispensables, il est difficile d'éviter quelques frictions. Les relations avec Bruxelles sont importantes: par le biais des fonds structurels, la Communauté Européenne intervient massivement dans trois domaines: le logement, la circulation et l'environnement, sans compter de multiples autres aides. On imagine donc aisément la complexité de certains dossiers.

\section{VII - UNE REDÉFINITION URBANISTIQUE DE L'ENSEMBLE DE LA VILLE}

Lisbonne est donc aujourd'hui une ville en pleine métamorphose. Les actions des différentes entités publiques se conjuguent à travers un faisceau de réalisations pour provoquer une mutation extrêmement rapide. Ce mouvement se caractérise par une réhabilitation rapide sous l'impulsion du déblocage progressif des loyers, de l'enrichissement d'une population souvent propriétaire de son appartement ${ }^{14}$, d'un important programme municipal de réhabilitation du bâti ancien déjà bien engagé. S'y ajoutent de multiples initiatives qui débouchent soit sur la réhabilitation soit sur la rénovation et les nombreux aménagements de voirie qui délogent les cabanes des friches réservées, parfois depuis très longtemps, à la construction des autoroutes. Comme souvent, ce sont d'ailleurs les friches appartenant aux diverses entités de l'Etat qui sont les plus occupées. S'y ajoutent aussi, à Lisbonne, du moins dans sa périphérie, certaines des immenses réserves foncières constituées par les compagnies immobilières. Le mouvement de desserrement de l'agglomération engagé depuis plus de quinze ans (RODRIGUES, 1994) se traduit par l'éviction de nombre de ces barracas de la proche banlieue. Sous l'impulsion des pouvoirs publics, à tous niveaux, et avec l'aide presqu'omniprésente des fonds de l'Union Européenne divers programmes ont donc été lancés. S'il n'est pas dans le projet de cet article de les détailler, il demeure que, comme en matière de politiques publiques, on doit les présenter afin de garder à l'esprit que les réalisation à destination de l'habitat précaire s'inscrivent dans un contexte large de requalification globale de la ville:

- S'agissant des constructions sauvages, une série de lois $^{15}$ a initié une rénovation du cadastre bientôt achevée. Cela permet dorénavant de mieux contrôler la

13 Ainsi l'Expresso du 17 Maio 97 recense en matière de planification pour la seule année 96 et le début 97:

- Alteração da Lei de Delimitação dos Sectores.

- Transferência para as Autarquias de Novas Competências e Recursos Financeiros.

14 Tendance qu'on s'efforce de favoriser encore pour les jeunes et les classes moyennes.

15 Portaria 243/84; decreto lei 804/87; on impose aussi la conception de planos de recuperação pour chaque concelho. L'ensemble forme ainsi la base légale sur laquelle s'appuie aussi le PER. 
construction de nouveaux édifices et de régulariser celle des édifices déjà construits ${ }^{16}$ pour peu qu'ils bénéficient de l'important effort conjoint d'équipement lancé depuis une dizaine d'années par les services publics (eau, électricité et égouts, plus récemment gaz et téléphone). Ainsi, alors même que la construction de la CRIL grignote peu à peu les barracas du Bairro do Prior Velho (Loures) la compagnie du téléphone équipe le quartier ${ }^{17}$.

Cet exemple laisse cependant planer des doutes sur la capacité de coordination de certains projets évoqués plus loin et montre à nouveau l'ambiguïté des catégories mentales et statistiques sur lesquelles nous bâtissons nos jugements: qu'est-ce qu'un bidonville? Cela explique aussi pour partie le décalage entre les données du recensement et celles de l'IGAPHE.

Non dénué d'arrière-pensées fiscales, cet effort cadastral vise surtout les grands ensembles périphériques, parfois gigantesques, mais il s'intéresse aussi à l'habitat de petite taille. Seront donc concernées à la fois les barracas des zones urbaines mais aussi les constructions de villégiature des périphéries: dans le concelho de Sesimbra, spectaculaires, les villas abandonnées inachevées sur la route du Cabo d'Espichel ou celles détruites de Portinho da Arrabida montrent que cette volonté de reprise en main est bien réelle. La figure 1 montre l'ampleur de la tâche. C'est pourtant, avec les efforts en matière de réseaux de circulation, un des domaines où la situation a le plus évolué durant la première moitié des années 90 . On peut donc supposer qu'avant la fin du siècle l'urbanisation clandestine aura disparu dans l'agglomération lisboète.

- Pour la rénovation du bâti ancien, le programme municipal lancé à Lisbonne vise à rénover $10 \%$ des bâtiments à l'aide de subventions qui amplifient l'effort des propriétaires. Cette estimation correspond à l'évaluation proposée sur la carte de la figure 3. Ce programme, échelonné de 93 à 98, ne concerne pas seulement les façades: installation du gaz de ville, de salles de bains, mise aux normes de l'habitat. Ambitieux, il semble cependant en bonne voie et se double de facilités de l'INH mais aussi de la Caixa Geral de Depósitos afin de favoriser l'accession à la propriété dans certains quartiers du centre sensibles à la spéculation (Alfama, Bairro Alto, Lapa par exemple mais aussi, moins connus, Estefânia ou Campo de Ourique).

Dans les périphéries, le même mouvement vise à réhabiliter, sous impulsion municipale, les centres anciens, au demeurant assez réduits, et les quais. Achevé ou en voie d'achèvement à Cascais, Oeiras, Seixal, Montijo, Sesimbra, Setúbal, Palmela, ce mouvement s'étend aujourd'hui à la moindre freguesia: Queluz (Sintra), Alhos Vedros (Moita) et même l'emblématique Sacavém (Loures). Il demeure que la ville-centre, Lisbonne, concentre l'essentiel de cet effort: la réhabilitation déjà évoquée des quartiers du centre est complétée par l'immense projet très anglo-saxon (waterfront) des quais lancé en 94 pour être achevé en 98.

- Ces efforts sont complétés par de nombreuses initiatives impliquées dans

16 Correio da Manhã, 8 Junho 95.

17 Diário de Notícias, 17 Junho 95. 
des programmes fort divers visant à requalifier l'environnement urbain: nous en retiendrons surtout la rénovation complète des quais et des friches industrielles. Mais on peut y ajouter les projets visant à maîtriser l'accroissement et la localisation des espaces tertiaires.

Principal programme: le remodelage et l'extension des réseaux de transport urbain: notamment le métro mais aussi les bus et certaines lignes des eléctricos, mise en place d'un réseau de transport ferroviaire à l'échelle de l'aire métropolitaine, construction du pont de Montijo, accroissement des débits du Pont du 25 Avril (et mise en place du transit ferroviaire), programme autoroutier afin de desserrer l'aire métropolitaine (PORTO, 1992). La diversité de cette énumération montre assez bien la volonté générale de moderniser la ville et prendre en compte une aire urbaine vaste.

- Enfin, outre ces efforts d'aménagement, la volonté de prendre en compte l'environnement et en même temps de préserver d'immenses espaces peu ou pas construits (Coroa Urbana de Transição à la limite du concelho de Lisbonne mais aussi au-delà dans l'espace métropolitain récent) ne sera pas sans influencer cette requalification de l'espace qui aiguise la spéculation immobilière. Il s'agit aussi de parvenir à éviter que cela n'accentue les ségrégations sociales et les excès.

A Lisbonne même, sont toujours concernés la colline de Monsanto et certains quartiers, ainsi entre Olivais et Chelas, volontairement sous-densifiés mais menacés. Hors de Lisbonne, le principe d'une ceinture verte à la britannique a bien été décidé depuis longtemps. Mais à la périphérie immédiate de la ville-centre (prolongeant Monsanto à l'ouest et rejoignant l'aéroport au nord) la décision de classer cette zone n'a pas été suivie de mesures conservatrices ni d'un projet d'aménagement bien concrets.

Aussi de nombreux journaux ${ }^{18}$ dénoncent-ils la poursuite des installations sauvages, les passe-droits et pressions des grands promoteurs qui s'étaient souvent constitués-là des réserves foncières considérables. Surtout, la rénovation du cadastre amène à régulariser la situation de certaines de ces pratiques. Les promoteurs ne sont pas seuls accusés: on dénonce aussi les utilisations contestables par les pouvoirs publics eux-mêmes de ces espaces pour leurs projets routiers mais aussi, dans les concelhos périphériques, les zones industrielles et commerciales ${ }^{19}$.

Au sud du Tage, l'ennemi c'est le mitage de plus en plus étendu de la pinède par une marée de villas (habitations principales ou secondaires). Le classement de la Serra d'Arrabida comme parc naturel ainsi que la présence (mais pour combien de temps?) de terrains militaires le long de la côte ou, ailleurs, l'éloignement et un réseau routier vite insuffisant ont préservé d'immenses espaces qui permettent à Lisbonne de disposer d'une véritable ceinture verte très peu peuplée mais plus loin que prévue (20 à $30 \mathrm{~km}$ du centre). Cette remarque est aussi particulièrement vraie au nord (nord du concelho de Sintra et l'intégralité de celui de Mafra par exemple).

18 Voir l'amusante promenade proposée par le Diário de Noticias, 13 Dezembro 94.

19 L'importante zone commerciale et industrielle d'Alfragide (Amadora), située au croisement des autoroutes pour Cascais, Sintra et du périphérique, est dans ce cas. 
N'oublions pas d'ajouter à ce poumon "vert" l'immense surface du Tage $\left(300 \mathrm{~km}^{2}\right.$ alors que le concelho de Lisbonne n'en mesure que 83!).

Pour une métropole de cette taille, les densités y sont aujourd'hui exceptionnellement basses vu leur proximité de l'agglomération dense. Il demeure que les progrès très rapides en matière de voies de communication laissent planer des menaces pour certaines zones: les autoroutes A8 et CREL au nord, le pont de Montijo au sud-est sont les principaux vecteurs de l'extension urbaine à venir dans ces zones, comme le sont déjà les autoroutes A2 vers Setubal ou A1 vers Santarém.

On voit ainsi que l'on fait feu de tout bois afin de réhabiliter, remodeler, moderniser, dynamiser Lisbonne, même si certaines de ces initiatives se révèlent contradictoires. Dans le cas qui nous concerne, les barracas, outre qu'elles bénéficient des retombées de bon nombre des projets précédents et de l'enrichissement au moins partiel de la population, un ambitieux programme spécifique est en cours.

\section{VIII - L'IMPORTANCE DU PROGRAMA ESPECIAL DE REALOJAMENTO (P.E.R.)}

Sur l'ensemble de l'aire métropolitaine le PER, déjà évoqué, s'efforce de rénover les barracas des aires métropolitaines de Lisbonne et Porto d'ici la fin du siècle. Les intentions sont louables, mais il s'agit aussi d'éviter de tomber dans le piège de l'apologie et d'en évaluer l'importance réelle. Un programme de plus après ceux (3) des années 80 , perdus dans les limbes dès l'inauguration des premières réalisations et responsables de quelques horreurs urbanistiques qui ne dépareraient pas les banlieues à problèmes de France ou Grande-Bretagne?

Non, ce plan tranche à la fois par son ampleur (rien qu'à Lisbonne, il concerne 100000 personnes) mais aussi par l'importance des moyens et des structures mis en œuvre. Par bien des aspects, ce programme rappelle la grande vague des Zones d'Urbanisation Prioritaires en France dans les années 60, bien qu'au Portugal les autorités locales y jouent un rôle majeur. Enfin, dans un pays où traditionnellement le logement est du ressort de l'initiative privée, ce qui a conduit à développer surtout les réalisations destinées aux nouvelles classes moyennes, une telle implication des pouvoirs publics est aussi un élément important qui ne sera pas sans conséquences à venir sur le marché immobilier.

Lancé en juin 93, il entre actuellement dans sa phase de réalisation et, sauf à Loures et Amadora, devrait s'achever dans les délais promis. Son financement est assuré pour moitié (les terrains ou des immeubles) par des dons de l'IGAPHE et pour l'autre moitié par des prêts à taux très faibles de l'INH. Les Câmaras Municipais contrôlent la réalisation du plan: elles ont la responsabilité de la planification, de la démolition des barracas, de la construction des nouveaux logements et de leur gestion.

Pour éviter la multiplication des grands ensembles et mieux intégrer ce plan dans l'ensemble des politiques d'aménagement locales, elles disposent de deux autres solutions. Elles peuvent ainsi acquérir des maisons sur le marché. Cela permet une meilleure insertion de ces populations fragiles dans l'ensemble de la ville 
et cela rejoint les politiques qui visent à réhabiliter le bâti ancien et, à Lisbonne, maintenir la population dans le concelho, voire à en attirer dans le centre pour éviter une tertiarisation trop importante. Cette solution est aujourd'hui celle dont la réalisation est la plus avancée (près de 15.000 foyers).

Une troisième solution existe qui, au moyen des Contratos de Desenvolvimento da Habitação $(\mathrm{CDH})$, permet de faire appel à l'initiative privée: des entrepreneurs s'engagent à construire un nombre déterminé de logements avec une garantie d'achat pour une part notable du programme. Il semble que ces projets démarrent lentement pour l'instant.

Le tout suppose aussi un minimum de concertation afin que certaines communes du centre ne cherchent à refouler une population peu valorisante en périphérie. Certains accusent aussi le gouvernement de se débarrasser sur les concelhos d'un parc immobilier dont la propriété pourrait se révéler plus tard un cadeau empoisonné. Aussi, pour ces trois types de relogement, l'appartement appartiendra à la famille au bout de quinze ans de location en accession à la propriété.

On a vu que tous les concelhos sont concernés mais ne s'y engagent pas avec la même énergie: entre 30 et 40000 logements (figure 5), soit plus de $3 \%$ du nombre total de logements, sont concernés (carte 4), 4,75\% (plus de 11000 logements) à Lisbonne voire 5,8 à Amadora, 6,4 à Oeiras mais seulement 2,4\% à Montijo, 2,3\% à Vila Franca, et même 1,9\% à Sintra.

L'ambition du programme de rénovation explique aussi que s'engage une réflexion sur les qualités urbanistiques de cet habitat nouveau à créer afin d'éviter de reproduire certaines erreurs des banlieues des grandes métropoles européennes $^{20}$, déjà ébauchées dans les premiers programmes lancés ces dernières années à Chelas surtout, mais aussi à Pedreira dos Húngaros ou Alto do Lumiar.

Le débat, s'il n'a pas encore débouché sur des réalisations, a au moins le mérite d'exister. On propose aussi de reprendre la solution utilisée dans le Bairro da Liberdade: $30 \%$ des familles ont pu disposer dans des conditions intéressantes d'une somme équivalente au prix d'un appartement social afin de construire ou acheter leur propre logement. Cette solution, malgré quelques scandales, plaît à la population et intéressait le très libéral gouvernement de monsieur Cavaco Silva tout en débarrassant la CML (Câmara Municipal de Lisboa) d'un problème épineux. La difficulté de mise en œuvre à grande échelle d'un tel projet et le changement de majorité politique ont pour l'instant retardé son application.

Le rôle des pouvoirs publics reste dominant. Parfois le Président de la Câmara Municipal n'hésite pas à imposer 300 logements sociaux dans un quartier peuplé de classes moyennes provoquant d'ailleurs une levée de boucliers des administrés électeurs (ainsi à Alto da Loba, freguesia de Paço de Arcos, à Oeiras).

S'ajoute la volonté d'adjoindre à ces réalisations un accompagnement social systématique dont l'efficacité sera à vérifier sur la durée. On clame dans tous les

20 Expresso, 17 Junho 95: Um gueto ou uma solução?

Diário de Notícias, 17 Junho 95: Erradicação de barracas pode criar novos guetos. 
services concernés le désir d'éviter les surdensifications, les trop grands ensembles, les trop hauts immeubles et de privilégier une insertion dans le maillage du bâti, au demeurant souvent assez lâche sur l'ensemble de l'aire métropolitaine. Il reste à savoir si ces intentions ne seront pas débordées par l'ampleur du programme, la spéculation foncière ou les contraintes financières: beaucoup de risques pour d'excellentes intentions.

\section{IX - DES IMPLICATIONS POLITIQUES IMPORTANTES}

Enfin, les implications politiques du projet ne sont pas à négliger. A rebours d'un discours souvent entendu sur le désengagement de l'Etat, cela marque le retour du politique dans la cité. Mais au profit des entités locales plutôt que du pouvoir central. On a déjà évoqué le désir des municipalités, particulièrement celle de Lisbonne, de mieux contrôler la production de l'espace dans la ville à travers le renouveau de la planification. Leur rôle est renforcé par les progrès de la législation. De plus, elles possèdent une part importante des espaces non construits de leur territoire. Le recul des emprises appartenant à l'Etat, très inégal selon les concelhos, va en accroître l'importance relative. Plus important, par le biais de l'IGAPHE, sur l'ensemble du pays, mais surtout à Lisbonne, plus de la moitié des 34.000 appartements que possède l'Etat avait déjà été transférée aux municipalités fin 95. Même si certaines accusent leur donateur de se débarrasser d'immeubles dégradés, c'est considérable.

Or, les municipalités sont déjà propriétaires d'un parc immobilier important: selon les services d'urbanisme de la Câmara Municipal de Lisboa, les municipalités seraient partout les principaux propriétaires surtout car elles gèrent déjà une grande partie de l'habitat social existant. Cette part va s'accroître. Cela peut contribuer à renforcer leur emprise sur leur territoire, déjà importante dans d'autres domaines, à deux conditions:

- que les contraintes particulières de ce type d'habitat ne se révèlent pas trop fortes et paralysantes;

- que cesse le mouvement de désengagement de l'Etat dans de nombreux domaines, souvent liés au social.

Une évolution des pouvoirs dans la ville est prévisible et déjà même bien engagée. Il apparait cependant difficile d'imaginer encore leurs stratégies.

On a évoqué aussi plus haut la nécessité pour les différents concelhos de travailler en concertation. Cela devrait amener le développement d'une coopération à l'échelle de la métropole qui jusqu'à présent était limitée. Mais celle-ci s'avère nécessaire: le recul de la population de Lisbonne et les progrès de ses périphéries amènent les plus puissants concelhos (notamment Loures ou Sintra), disposant d'espace et de population abondants, polarisant une part croissante de l'activité économique, à se positionner comme des interlocuteurs incontournables. Une coopération indispensable à la gestion d'une métropole moderne qui se découvre des ambitions. 
Une nouveauté donc, dans un pays où la puissance des municipalités est réellement importante et symbolise le retour de la démocratie. Que cela soit possible dans ce domaine difficile serait de bon augure pour d'autres coopérations. La coordination des projets en matière de transport a aussi permis une première ébauche de rapprochement.

On évoque donc avec une insistance croissante la création d'une structure qui donnerait à l'Area Metropolitana de Lisboa, à vocation surtout statistique, des prérogatives plus importantes. Cette tendance à la redéfinition des cadres spatiaux urbains est générale dans les pays développés. De nombreuses études montrent le développement des coopérations (LEFEVRE et JOUVE, 1992) et l'adaptation, nécessairement un peu plus lente, des cadres administratifs et politiques (BOOTH, 1991; BARNEZET, 1993; PETERSON, 1981; OTTENSMANN, 1996; AMES et BROWN, 1992). Débutée tardivement à Lisbonne, cette évolution semble inéluctable.

\section{X - CONCLURE: LA FIN DU ROMANTISME?}

A travers l'évolution de l'habitat précaire, particulièrement des barracas, nous avons essayé de montrer les métamorphoses que connaît Lisbonne. La rénovation actuelle, autant que la réhabilitation d'une partie du bâti ancien, s'inscrit dans un contexte plus global de modernisation.

Les barracas existent, c'est indéniable, la ville n'est pas toujours aussi propre et pimpante qu'on la rêverait, c'est certain. Cependant, les bidonvilles ne sont pas faciles à définir et importe beaucoup la vision qu'on en a, très souvent nord-européenne, mais au Portugal aussi le discours change. Si le pays est en net progrès, il reste le plus pauvre de la Communauté Européenne (INSEE, 1997); n'en attendons pas trop une perfection déjà bien difficile et illusoire en Europe du nord, une perfection d'homme riche sûr de son fait et de son pouvoir d'achat. Les conditions de vie réelles à Lisbonne sont bien meilleures que ce qu'on pourrait supposer à la lecture du PIB par habitant. Surtout, l'énorme retard accumulé ces dernières décennies sera finalement rattrapé en un délai bien plus court.

Les barracas devraient sinon totalement disparaître du moins se raréfier dans les années qui viennent. La réhabilitation du bâti existant est bien engagée et, la spéculation foncière aidant, fera disparaître une image de Lisbonne déjà surfaite. C'était d'ailleurs l'enjeu de l'accélération des réhabilitations en 1994 lorsque Lisbonne fut capitale européenne de la culture, c'est encore celui de Expo'98.

Si l'on se rappelle que l'aire métropolitaine se signale aussi par une relativement faible densification, l'importance des petits immeubles et souvent des maisons particulières, on peut donc, malgré les barracas, garder une image moins pessimiste de la qualité de vie pour la plupart des habitants de la métropole que celle qui apparait dans beaucoup de discours. Qu'il demeure des problèmes est évident mais la qualité de l'habitat devrait continuer de s'améliorer rapidement.

Surtout, la principale évolution est politique avec un retour marqué de l'action publique dans un domaine longtemps négligé. Outre que cela renforce le pouvoir et 
accroît la crédibilité des municipalités, on peut en attendre un renforcement des contrôles qui limiteront le déplacement ou la réapparition du phénomène.

Enfin, pour le chercheur, Lisbonne présente la particularité intéressante d'être une ville qui évolue très vite: un "laboratoire urbain" chaque jour plus proche des autres métropoles de la Communauté Européenne. C'est particulièrement clair pour le géographe ou l'urbaniste impressionné par le renouveau de la planification et l'ampleur comme la rapidité de la redéfinition urbaine. Mais c'est aussi sensible jusque dans les comportements les plus intimes des habitants de la métropole. La démographie est un exemple de changement radical souvent mentionné mais ce sont aussi les travers que connaissent les cités du nord: éclatement de la vie des quartiers, montée du sentiment d'insécurité, mitage périurbain, utilisation sans cesse croissante de l'automobile, multiplication rapide des centres commerciaux de périphérie et fuite massive les week-end.

\section{BIBLIOGRAPHIE}

AMES, D.L., N. C. BROWN et al (1992) - Rethinking American urban policy. Journal of Urban Affairs, 14: 197-216.

BARNEZET, S. (1993) - Réforme du code de l'urbanisme. Projet de loi de Jean-Louis Bianco. Territoires, 334. Paris, p. 59.

BASTIE, J., B. DeZert (1991) - La ville. Paris, Masson, chap. 29, p. 349 et sq.

Воотн, Рн. (1991) - L'évolution du rapport central-local en Grande-Bretagne. Actes du colloque de Royaumont, ADEF. Paris:43-58.

Bruno SoARes, L. (1984) - Urbanização Clandestina e Política Urbana. Sociedade e Território $\mathrm{n}^{\circ}$ 1:19-34.

BRUNo SoAReS, L. (1994) - Lisboa - As Bases do Novo Planeamento da Cidade (1990-94). Finisterra - Revista Portuguesa de Geografia, XXIX (57):147-156

CACHINHO, H. (1994) - O comércio a retalho na cidade de Lisboa. Reestruturação económica e dinâmicas territoriais. Finisterra - Revista Portuguesa de Geografia, XXIX, (57): 119-144.

CASTELls, M. (1981) - La question urbaine, Paris, Maspero, 526 p.

Daveau, S. (1989) - Geografia de Portugal, III. O Povo Português. Lisboa, Ed. João Sá Da Costa Lda, $1^{\text {a }}$ ed: 898-899.

DiÁRIO DE NOTICIAS, 17 Junho 95: Erradicação de barracas pode criar novos guetos.

DRAIN, M. (1990) - Le Portugal. in Géographie Universelle, sous la direction de R. BRUNET, Tome 1, livre Second. Paris. Hachette / Reclus: 283

EXPRESSO, 17 Maio 1997, Cadastro único pronto em 1998.

FONSECA, M.L. (1988) - Lisbonne depuis un siècle: de la cité à l'aire métropolitaine. Revue géographique des Pyrénées et du sud-ouest. Toulouse, 2-3, (59): 187-204.

FranCE. MinISTERE DE L'INTERIEUR. DIRECTION GENERALE DES COMMUNAUTES LOCALES (DGCL) (1992) - Loi Joxe, la démocratie locale en trois points. Territoires, 327. Paris, p. 8.

GEORGE, P. (1961) - Précis de géographie urbaine. Paris, PUF, $1^{\mathrm{e}}$ ed. $289 \mathrm{p}$.

Guerra, I.; P. CostA, T. et M. JoÃo QueIDAS (1995) - Appropriation du logement et modes de vie: "Clandestins" et "Légaux". Espaces et sociétés, 79: Modes de vie et 
société portugaise, Paris, L'Harmattan: 7-25.

Guichard, F. (1990) - Géographie du Portugal. Paris, Masson. 224 p.

INSEE (1997) - La pauvreté dans la Communauté Européenne en 1993, INSEE-Première, 482, Paris.

LEFEVRE, C., B. Jouve (1992) - Nouveaux acteurs et nouveaux territoires dans les grandes agglomérations européennes: une approche comparative à partir des réseaux lourds de transports collectifs. Annales de Géographie, 568, Paris: 622-652.

LOBO, I. S. (1986) - Construção Não-Formal - Contribução para a Análise Quantitativa a Nivel Regional. Lisboa, IACEP-NEUR, Estudos Urbanos e Regionais, 36.

LOUREIRO DE MATOS, F. (1990) - A construção clandestina em Vila Nova de Gaia - o caso dos bairros do Picão e da Madalena Nascente. Revista da Faculdade de Letras-Geografia, Porto, vol. VI: 149-280.

Ministério do Equipamento Social, Gabinete de Estudos e Planeamento da HABITAÇÃo E OBRAS PÚBliCAS (1984) - A Situação Habitacional Segundo o Recenseamento de 1981. Lisboa.

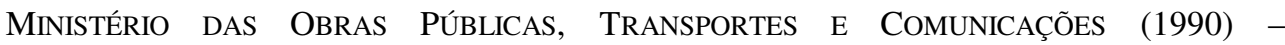
Características Recentes do Sector da Construção. Lisboa

Ottensmann, J.R. (1996) - The New Central Cities. Implications of the New Definition of the Metro-politan Area. Urban Affairs Review, 31, (5): 681-691.

PETERSON, P.E. (1981) - City limits. Chicago, University of Chicago Press.

PorTo, M. L. (1992) - A localização do Novo Aeroporto de Lisboa e sua Articulação com os demais Meios de Transporte. Lisboa, Centro de Estudos Geográficos, 25 p.

RODRIGUES, M. J. (1994) - Alguns dados estatísticos sobre a área metropolitana de Lisboa. Finisterra - Revista Portuguesa de Geografia, XXIX (57): 185-195.

SAlgueIRO, T. B. (1972) - Bairros Clandestinos na Periferia de Lisboa. Lisboa, C.E. G: 2

SAlgueiro, T. B. (1977) - Bairros Clandestinos na Periferia de Lisboa. Finisterra Revista Portuguesa de Geografia, XII (23): 28-55.

SALGUEIRO, T. B. (1994) - Novos produtos imobiliários e reestruturação urbana. Finisterra - Revista Portuguesa de Geografia, XXIX (57):79-101.

TomAs, F. (1993) - Séminaires Formes urbaines, DEA Villes et Sociétés, Université Lyon II Lumière. Non publié. 